\title{
The Sharp Bound of the Hankel Determinant of the Third Kind for Starlike Functions of Order $1 / 2$
}

\section{Adam Lecko $^{1}$ D Y Young Jae Sim $^{2} \cdot$ Barabara Śmiarowska $^{1}$}

Received: 22 November 2017 / Accepted: 28 June 2018 / Published online: 4 July 2018

(c) The Author(s) 2018

\section{Abstract}

In the present paper, we proved the sharp inequality $\left|H_{3,1}(f)\right| \leq 1 / 9$ for analytic functions $f$ with $a_{n}:=f^{(n)}(0) / n !, n \in \mathbb{N}, a_{1}:=1$, such that

$$
\operatorname{Re} \frac{z f^{\prime}(z)}{f(z)}>\frac{1}{2}, \quad z \in \mathbb{D}:=\{z \in \mathbb{C}:|z|<1\}
$$

where

$$
H_{3,1}(f):=\left|\begin{array}{lll}
a_{1} & a_{2} & a_{3} \\
a_{2} & a_{3} & a_{4} \\
a_{3} & a_{4} & a_{5}
\end{array}\right|
$$

is the third Hankel determinant.

Keywords Starlike functions of order 1/2 · Carathéodory functions . Hankel determinant . Coefficients

Mathematics Subject Classification 30C45

Communicated by David Shoikhet.

$\triangle$ Adam Lecko

alecko@matman.uwm.edu.pl

Young Jae Sim

yjsim@ks.ac.kr

Barabara Śmiarowska

b.smiarowska@matman.uwm.edu.pl

1 Department of Complex Analysis, Faculty of Mathematics and Computer Science,

University of Warmia and Mazury in Olsztyn, ul. Słoneczna 54, 10-710 Olsztyn, Poland

2 Department of Mathematics, Kyungsung University, Busan 48434, Korea

Birkhäuser 


\section{Introduction}

Let $\mathcal{H}$ be the class of analytic functions in $\mathbb{D}:=\{z \in \mathbb{C}:|z|<1\}$ and let $\mathcal{A}$ be its subclass of functions $f$ normalized by $f(0):=0, f^{\prime}(0):=1$, i.e., of the form

$$
f(z)=\sum_{n=1}^{\infty} a_{n} z^{n}, \quad a_{1}:=1, z \in \mathbb{D} .
$$

Given $\alpha \in[0,1)$, let $\mathcal{S}^{*}(\alpha)$ denote the subclass of $\mathcal{A}$ of functions $f$ such that

$$
\operatorname{Re} \frac{z f^{\prime}(z}{f(z)}>\alpha, \quad z \in \mathbb{D},
$$

called starlike of order $\alpha$. In particular, $\mathcal{S}^{*}(0)=: \mathcal{S}^{*}$ is the class of starlike functions, i.e., the family of all univalent functions in $\mathcal{A}$ which map $\mathbb{D}$ onto starlike domains (with respect to the origin). Starlike functions of order $\alpha$ were introduced by Robertson [19] (see also [7, Vol. I, p. 138]). An important role is played by the class $\mathcal{S}^{*}(1 / 2)$. One of the significant results belongs to Marx [15] and Strohhäcker [23]. They proved that

$$
\mathcal{S}^{c} \subset \mathcal{S}^{*}(1 / 2)
$$

(see also [16, Theorem 2.6a, p. 57]), where $\mathcal{S}^{c}$ means the class of convex functions, i.e., the family of all univalent functions in $\mathcal{A}$ which map $\mathbb{D}$ onto convex domains. By the well known result due to Study ([24], see also [6, p. 42]) a function $f$ is in $\mathcal{S}^{c}$ if and only if

$$
\operatorname{Re}\left\{1+\frac{z f^{\prime \prime}(z)}{f^{\prime}(z)}\right\}>0, \quad z \in \mathbb{D} \text {. }
$$

What is interesting, a function

$$
f(z):=\frac{z}{1-z}, \quad z \in \mathbb{D}
$$

is extremal for many computational problems in both these two classes, i.e., in $\mathcal{S}^{c}$ and $\mathcal{S}^{*}(1 / 2)$.

For $q, n \in \mathbb{N}$, the Hankel determinant $H_{q, n}(f)$ of function $f \in \mathcal{A}$ of the form (1.1) is defined as

$$
H_{q, n}(f):=\left|\begin{array}{cccc}
a_{n} & a_{n+1} & \cdots & a_{n+q-1} \\
a_{n+1} & a_{n+2} & \cdots & a_{n+q} \\
\vdots & \vdots & \vdots & \vdots \\
a_{n+q-1} & a_{n+q} & \cdots & a_{n+2(q-1)}
\end{array}\right| .
$$

Given a subfamily $\mathcal{F}$ of $\mathcal{A}, q$ and $n$, computing the upper bound of $H_{q, n}$ is an interesting problem to study. Recently many authors examined the Hankel determinant $H_{2,2}(f)=$ 
$a_{2} a_{4}-a_{3}^{2}$ of order 2 (see e.g., $[4,5,8,9,12,17]$ ). Note also that $H_{2,1}(f)=a_{3}-a_{2}^{2}$ is the well known coefficient functional which for $\mathcal{S}$ was estimated in 1916 by Bieberbach (see e.g., [7, Vol. I, p. 35]). To find the upper bound of the Hankel determinant

$$
H_{3,1}(f)=\left|\begin{array}{lll}
a_{1} & a_{1} & a_{3} \\
a_{2} & a_{3} & a_{4} \\
a_{3} & a_{4} & a_{5}
\end{array}\right|=a_{3}\left(a_{2} a_{4}-a_{3}^{2}\right)-a_{4}\left(a_{4}-a_{2} a_{3}\right)+a_{5}\left(a_{3}-a_{2}^{2}\right)
$$

of the third kind, is more difficult if we expect to get sharp estimate. Results in this direction however not sharp were obtained by various authors, e.g., [1,2,4,5,20-22,25].

In this paper, we found the sharp bound of the Hankel determinant $H_{3,1}$ over the class $\mathcal{S}^{*}(1 / 2)$, namely, we proved that $\left|H_{3,1}(f)\right| \leq 1 / 9$ for $f \in \mathcal{S}^{*}(1 / 2)$ and that the inequality is sharp. Since the class $\mathcal{S}^{*}(1 / 2)$ has a representation with using the Carathéodory class $\mathcal{P}$, i.e., the class of functions $p \in \mathcal{H}$ of the form

$$
p(z)=1+\sum_{n=1}^{\infty} c_{n} z^{n}, \quad z \in \mathbb{D}
$$

having a positive real part in $\mathbb{D}$, the coefficients of functions in $\mathcal{S}^{*}(1 / 2)$ have a suitable representation expressed by coefficients of functions in $\mathcal{P}$. Therefore to get the upper bound of $H_{3,1}$, we based our computing on the well known formulas on coefficient $c_{2}$ (e.g., [18, p. 166]), the formula $c_{3}$ due to Libera and Zlotkiewicz $[13,14]$ and the formula for $c_{4}$ recently found in [11].

At the end let us mention that in [10] the authors proved that $\left|H_{3,1}(f)\right| \leq 4 / 135=$ $0.0296 \ldots$ for $f \in \mathcal{S}\rfloor$ and that the result is sharp. Looking at the inclusion (1.3) we can state that the the corresponding bounds of $H_{3,1}$ carry some information about the richness of classes. Classical estimates of coefficients does not necessarily include such a distinction, e.g., both in the class $\mathcal{S}^{c}$ and in the class $\mathcal{S}^{*}(1 / 2)$ modules of all coefficients are bounded by 1 (see [7, Theorem 7, p. 117; Theorem 2, p. 140]) with the extremal function given by (1.4).

\section{Main Result}

The basis for proof of the main result is the following lemma which contains the well known formula for $c_{2}$ (e.g., [18, p. 166]), the formula for $c_{3}$ due to Libera and Zlotkiewicz $[13,14]$ and the formula for $c_{4}$ found in [11].

Lemma 2.1 If $p \in \mathcal{P}$ is of the form (1.6) with $c_{1} \geq 0$, then

$$
\begin{aligned}
& 2 c_{2}=c_{1}^{2}+\left(4-c_{1}^{2}\right) \zeta \\
& 4 c_{3}=c_{1}^{3}+\left(4-c_{1}^{2}\right) c_{1} \zeta(2-\zeta)+2\left(4-c_{1}^{2}\right)\left(1-|\zeta|^{2}\right) \eta
\end{aligned}
$$


and

$$
\begin{aligned}
8 c_{4}= & c_{1}^{4}+\left(4-c_{1}^{2}\right) \zeta\left[c_{1}^{2}\left(\zeta^{2}-3 \zeta+3\right)+4 \zeta\right] \\
& -4\left(4-c_{1}^{2}\right)\left(1-|\zeta|^{2}\right)\left[c_{1}(\zeta-1) \eta+\bar{\zeta} \eta^{2}-\left(1-|\eta|^{2}\right) \xi\right]
\end{aligned}
$$

for some $\zeta, \eta, \xi \in \overline{\mathbb{D}}:=\{z \in \mathbb{C}:|z| \leq 1\}$.

We will now estimate the third order Hankel determinant $H_{3,1}(f)$ for $f \in \mathcal{S}^{*}(1 / 2)$.

\section{Theorem 2.2}

$$
\max \left\{\left|H_{3,1}(f)\right|: f \in \mathcal{S}^{*}(1 / 2)\right\}=\frac{1}{9}
$$

with the extremal function

$$
f(z):=\frac{z}{\sqrt[3]{1-z^{3}}}, \quad z \in \mathbb{D}, \sqrt[3]{1}:=1
$$

Proof Let $f \in \mathcal{S}^{*}(1 / 2)$ be of the form (1.1). Then by (1.2) we have

$$
z f^{\prime}(z)=\frac{1}{2}(p(z)+1) f(z), \quad z \in \mathbb{D}
$$

for some function $p \in \mathcal{P}$ of the form (1.6). Since the classes $\mathcal{P}$ and $\mathcal{S}^{*}(1 / 2)$ are invariant under the rotations, by Carathéodory Theorem we may assume that $c:=$ $c_{1} \in[0,2]$ ([3], see also [7, Vol. I, p. 80, Theorem 3]). Putting the series (1.1) and (1.6) into (2.6) and equating coefficients we get

$$
\begin{aligned}
& a_{2}=\frac{1}{2} c, \quad a_{3}=\frac{1}{8}\left(2 c_{2}+c^{2}\right), \quad a_{4}=\frac{1}{48}\left(8 c_{3}+6 c c_{2}+c^{3}\right), \\
& a_{5}=\frac{1}{384}\left(48 c_{4}+32 c c_{3}+12 c_{2}^{2}+12 c^{2} c_{2}+c^{4}\right) .
\end{aligned}
$$

Hence and by (1.5) we have

$$
\begin{aligned}
H_{3,1}(f)= & \frac{1}{9216}\left(-c^{6}+6 c^{4} c_{2}-72 c_{2}^{3}+32 c^{3} c_{3}\right. \\
& \left.+192 c c_{2} c_{3}-256 c_{3}^{2}-36 c^{2} c_{2}^{2}+288 c_{2} c_{4}-144 c^{2} c_{4}\right) .
\end{aligned}
$$

To simplify computation, let $t:=4-c^{2}$. Thus formulas (2.1)-(2.3) we can rewrite as

$$
\begin{aligned}
c_{2}= & \frac{1}{2}\left(c^{2}+t \zeta\right), \quad c_{3}=\frac{1}{4}\left(c^{3}+2 c t \zeta-c t \zeta^{2}+2 t\left(1-|\zeta|^{2}\right) \eta\right), \\
c_{4}= & \frac{1}{8}\left[c^{4}+3 c^{2} t \zeta+\left(4-3 c^{2}\right) t \zeta^{2}+c^{2} t \zeta^{3}+4 t\left(1-|\zeta|^{2}\right)\left(c \eta-c \zeta \eta-\bar{\zeta} \eta^{2}\right)\right. \\
& \left.+4 t\left(1-|\zeta|^{2}\right)\left(1-|\eta|^{2}\right) \xi\right] .
\end{aligned}
$$


Hence by straightforward algebraic computation we have

$$
\begin{aligned}
6 c^{4} c_{2}= & 3\left(c^{6}+c^{4} t \zeta\right), \\
72 c_{2}^{3}= & 9\left[c^{6}+3 c^{4} t \zeta+3 c^{2} t^{2} \zeta^{2}+t^{3} \zeta^{3}\right] \\
32 c^{3} c_{3}= & 8\left[c^{6}+2 c^{4} t \zeta-c^{4} t \zeta^{2}+2 c^{3} t\left(1-|\zeta|^{2}\right) \eta\right], \\
192 c c_{2} c_{3}= & 24\left[c^{6}+3 c^{4} t \zeta+2 c^{2} t^{2} \zeta^{2}-c^{4} t \zeta^{2}-c^{2} t^{2} \zeta^{3}\right. \\
& \left.+2 t\left(c^{3}+c t \zeta\right)\left(1-|\zeta|^{2}\right) \eta\right] \\
256 c_{3}^{2}= & 16\left[c^{6}+4 c^{4} t \zeta+4 c^{4} t^{2} \zeta^{2}-2 c^{4} t \zeta^{2}-4 c^{2} t^{2} \zeta^{3}+c^{2} t^{2} \zeta^{4}\right. \\
& \left.+4 t\left(c^{3}+2 c t \zeta-c t \zeta^{2}\right)\left(1-|\zeta|^{2}\right) \eta+4 t^{2}\left(1-|\zeta|^{2}\right)^{2} \eta^{2}\right], \\
36 c^{2} c_{2}^{2}= & 9\left[c^{6}+2 c^{4} t \zeta+c^{2} t^{2} \zeta^{2}\right], \\
144\left(2 c_{2} c_{4}-c^{2} c_{4}\right)= & 18\left[c^{4} t \zeta+3 c^{2} t^{2} \zeta^{2}+\left(4-3 c^{2}\right) t^{2} \zeta^{3}+c^{2} t^{2} \zeta^{4}\right. \\
& +4 t^{2} c \zeta(1-\zeta)\left(1-|\zeta|^{2}\right) \eta \\
& \left.-4 t^{2}\left(1-|\zeta|^{2}\right)|\zeta|^{2} \eta^{2}+4 t^{2}\left(1-|\zeta|^{2}\right)\left(1-|\eta|^{2}\right) \zeta \xi\right] .
\end{aligned}
$$

Setting the above expression to (2.7) we get

$$
\begin{aligned}
& H_{3,1}(f) \\
& =\frac{1}{9216}\left(4-c^{2}\right)^{2}\left[\gamma_{1}(c, \zeta)+\gamma_{2}(c, \zeta) \eta+\gamma_{3}(c, \zeta) \eta^{2}+\gamma_{4}(c, \zeta, \eta) \xi\right],
\end{aligned}
$$

where for $\zeta, \eta, \xi \in \overline{\mathbb{D}}$,

$$
\begin{aligned}
& \gamma_{1}(c, \zeta):=\zeta^{2}\left[2 c^{2}+\left(36-5 c^{2}\right) \zeta+2 c^{2} \zeta^{2}\right] \\
& \gamma_{2}(c, \zeta):=-8 c \zeta(1+\zeta)\left(1-|\zeta|^{2}\right) \\
& \gamma_{3}(c, \zeta):=-8\left(8+|\zeta|^{2}\right)\left(1-|\zeta|^{2}\right)
\end{aligned}
$$

and

$$
\gamma_{4}(c, \zeta, \eta):=72\left(1-|\zeta|^{2}\right)\left(1-|\eta|^{2}\right) \zeta
$$

Let $x:=|\zeta| \in[0,1]$ and $y:=|\eta| \in[0,1]$. Since $|\xi| \leq 1$, from (2.8) we obtain

$$
\begin{aligned}
\left|H_{3,1}(f)\right| \leq & \frac{1}{9216}\left(4-c^{2}\right)^{2}\left[\left|\gamma_{1}(c, \zeta)\right|\right. \\
& \left.+\left|\gamma_{2}(c, \zeta)\right||\eta|+\left|\gamma_{3}(c, \zeta)\right||\eta|^{2}+\left|\gamma_{4}(c, \zeta, \eta)\right|\right] \\
\leq & \frac{1}{9216}\left(4-c^{2}\right)^{2} F(c, x, y),
\end{aligned}
$$


where

$$
F(c, x, y):=f_{1}(c, x)+f_{4}(c, x)+f_{2}(c, x) y+\left(f_{3}(c, x)-f_{4}(c, x)\right) y^{2},
$$

with

$$
\begin{aligned}
& f_{1}(c, x):=x^{2}\left[2 c^{2}+\left(36-5 c^{2}\right) x+2 c^{2} x^{2}\right], \\
& f_{2}(c, x):=8 c x(1+x)\left(1-|x|^{2}\right), \\
& f_{3}(c, x):=8\left(8+x^{2}\right)\left(1-x^{2}\right)
\end{aligned}
$$

and

$$
f_{4}(c, x):=72\left(1-x^{2}\right) x .
$$

Now, we will show that

$$
F(c, x, y) \leq 64, \quad c \in[0,2], x \in[0,1], y \in[0,1] .
$$

Since $f_{2}(c, x)>0$ and

$$
f_{3}(c, x)-f_{4}(c, x)=8(1-x)(8-x)\left(1-x^{2}\right)>0
$$

for $c \in(0,2)$ and $x \in(0,1)$, so for $c \in(0,2)$ and $x \in(0,1)$,

$$
\begin{aligned}
F(c, x, y) \leq & F(c, x, 1) \\
= & f_{1}(c, x)+f_{2}(c, x)+f_{3}(c, x) \\
= & x^{2}(x-2)(2 x-1) c^{2}+8 x(x+1)\left(1-x^{2}\right) c \\
& -4\left(2 x^{4}-9 x^{3}+14 x^{2}-16\right)=: G(c, x) .
\end{aligned}
$$

For $x=1 / 2$ the function $(0,2) \ni c \mapsto G(c, 1 / 2)$ has no critical point, obviously. When $x \neq 1 / 2$, then $\partial G / \partial c=0$ iff

$$
c=\frac{4 x(x+1)\left(1-x^{2}\right)}{x^{2}(2-x)(2 x-1)}=: c_{0} \in(0,2),
$$

which holds only for $x \in((2+3 \sqrt{2}) / 7,1)$. Thus

$$
\frac{\partial G}{\partial x}\left(c_{0}, x\right)=0
$$

iff

$$
\begin{aligned}
& 4\left(8 x^{2}-15 x+4\right)(x+1)^{2}\left(1-x^{2}\right)^{2} \\
& \quad+8\left(4 x^{3}+3 x^{2}-2 x-1\right)(x+1)\left(1-x^{2}\right)(x-2)(2 x-1) \\
& -x^{2}\left(8 x^{2}-27 x+28\right)(x-2)^{2}(2 x-1)^{2}=0
\end{aligned}
$$


which after simplifying reduces to

$$
-64 x^{7}+320 x^{6}-788 x^{5}+1503 x^{4}-1624 x^{3}+760 x^{2}-80 x-36=0
$$

for $x \in((2+3 \sqrt{2}) / 7,1)$. As we can check the above equation has no solution in $((2+3 \sqrt{2}) / 7,1)$ (real solutions are $\left.x_{1} \approx-0.1513, x_{2} \approx 1.0622, x_{3} \approx 2.4952\right)$. Thus the function $G$ has no critical point in $(0,2) \times(0,1)$.

For $c=0$ and $c=2$ both functions

$$
g_{1}(x):=F(0, x, 1)=4\left(-2 x^{4}+9 x^{3}-14 x^{2}+16\right), \quad x \in[0,1],
$$

and

$$
g_{2}(x):=F(2, x, 1)=16\left(-x^{4}-2 x^{2}+4\right), \quad x \in[0,1]
$$

are decreasing, so

$$
g_{i}(x) \leq g_{i}(0)=64, \quad i=1,2, x \in[0,1] .
$$

For $x=0$ and $x=1$ we have respectively,

$$
F(c, 0,1)=64, \quad c \in[0,2],
$$

and

$$
F(c, 1,1)=-c^{2}+36 \leq 36, \quad c \in[0,2]
$$

Hence, by (2.12) and (2.11) it follows that the (2.9) holds. This together with (2.9) shows that $\left|H_{3,1}(f)\right| \leq 1 / 9$.

For the function (2.5) which is in $\mathcal{S}^{*}(1 / 2)$, we have $a_{2}=a_{3}=a_{5}=0$ and $a_{4}=1 / 3$. Thus $H_{3,1}(f)=-1 / 9$, which makes equality in (2.4).

Acknowledgements This work was partially supported by the National Research Foundation of Korea (NRF) grant funded by the Korea government (Ministry of Science, ICT and Future Planning) (No. NRF2017R1C1B5076778).

Open Access This article is distributed under the terms of the Creative Commons Attribution 4.0 International License (http://creativecommons.org/licenses/by/4.0/), which permits unrestricted use, distribution, and reproduction in any medium, provided you give appropriate credit to the original author(s) and the source, provide a link to the Creative Commons license, and indicate if changes were made.

\section{References}

1. Babalola, K.O.: On $\mathrm{H}_{3}$ (1) Hankel determinants for some classes of univalent functions. In: Cho, Y.J. (ed.) Inequality Theory and Applications, vol. 6, pp. 1-7. Nova Science Publishers, New York (2010)

2. Bansal, D., Maharana, S., Prajapat, J.K.: Third order Hankel determinant for certain univalent functions. J. Korean Math. Soc. 52(6), 1139-1148 (2015) 
3. Carathéodory, C.: Über den Variabilitatsbereich der Koeffizienten von Potenzreihen, die gegebene werte nicht annehmen. Math. Ann. 64, 95-115 (1907)

4. Cho, N.E., Kowalczyk, B., Kwon, O.S., Lecko, A., Sim, Y.J.: The bounds of some determinants for starlike functions of order alpha. Bull. Malays. Math. Sci. Soc. 41, 523-535 (2018)

5. Cho, N.E., Kowalczyk, B., Kwon, O.S., Lecko, A., Sim, Y.J.: The bound of the Hankel detrminant for strongly starlike functions of order alpha. J. Math. Inequal. 11(2), 429-439 (2017)

6. Duren, P.T.: Univalent Functions. Springer, New York (1983)

7. Goodman, A.W.: Univalent Functions. Mariner, Tampa (1983)

8. Janteng, A., Halim, S.A., Darus, M.: Coefficient inequality for a function whose derivative has a positive real part. J. Inequal. Pure Appl. Math. 7(2), 1-5 (2006). Art. 50

9. Janteng, A., Halim, S.A., Darus, M.: Hankel determinant for starlike and convex functions. Int. J. Math. Anal. 1(13), 619-625 (2007)

10. Kowalczyk, B., Lecko, A., Sim, Y.J.: The sharp bound of the Hankel determinant of the third kind for convex functions. Bull. Austr. Math. Soc. 97, 435-445 (2018)

11. Kwon, O.S., Lecko, A., Sim, Y.J.: On the fourth coefficient of functions in the Carathéodory class. Comput. Methods Funct. Theory 18, 307-314 (2018)

12. Lee, S.K., Ravichandran, V., Supramanian, S.: Bound for the second Hankel determinant of certain univalent functions. J. Inequal. Appl. 2013(281), 1-17 (2013)

13. Libera, R.J., Zlotkiewicz, E.J.: Early coefficients of the inverse of a regular convex function. Proc. Am. Math. Soc. 85(2), 225-230 (1982)

14. Libera, R.J., Zlotkiewicz, E.J.: Coefficient bounds for the inverse of a function with derivatives in $\mathcal{P}$. Proc. Am. Math. Soc. 87(2), 251-257 (1983)

15. Marx, A.: Untersuchungenúber schlichte Abbildungen. Math. Ann. 107, 40-65 (1932/33)

16. Miller, S.S., Mocanu, P.T.: Differential Subordinations. Theory and Applications. Marcel Dekker Inc, New York (2000)

17. Mishra, A.K., Gochhayat, P.: Second Hankel determinat for a class of analytic functions defined by fractional derivative. Int. J. Math. Math. Sci. 2008, 1-10 (2008). Article ID 153280

18. Pommerenke, C.: Univalent Functions. Vandenhoeck \& Ruprecht, Göttingen (1975)

19. Robertson, M.S.: On the theory of univalent functions. Ann. Math. 37, 374-408 (1936)

20. Prajapat, J.K., Bansal, D., Singh, A., Mishra, A.K.: Bounds on third Hankel determinant for close-toconvex functions. Acta Univ. Sapientae Math. 7(2), 210-219 (2015)

21. Raza, M., Malik, S.N.: Upper bound of the third Hankel determinant for a class of analytic functions related with lemniscate of Bernoulli. J. Inequal. Appl. 2013(412), 8 (2013)

22. Shanmugam, G., Stephen, B.A., Babalola, K.O.: Third Hankel determinant for $\alpha$-starlike functions. Gulf J. Math. 2(2), 107-113 (2014)

23. Strohhäcker, E.: Beitrage zur Theorie der schlichten Funktionen. Math. Z. 37, 356-380 (1933)

24. Study, E.: Vorlesungen über ausgewählte Gegenstände der Geometrie, Zweites Heft; Konforme Abbildung Einfach-Zusammenhängender Bereiche. Druck und Verlag von B.G. Teubner, Leipzig (1913)

25. Sudharsan, T.V., Vijayalaksmi, S.P., Sthephen, B.A.: Third Hankel determinant for a subclass of analytic functions. Malays. J. Math. 2(4), 438-444 (2014) 